PROCEEDINGS OF THE

AMERICAN MATHEMATICAL SOCIETY

Volume 132, Number 3, Pages 831-840

S 0002-9939(03)07101-6

Article electronically published on August 19, 2003

\title{
APPROXIMATE FIXED POINT SEQUENCES AND CONVERGENCE THEOREMS FOR LIPSCHITZ PSEUDOCONTRACTIVE MAPS
}

\author{
C. E. CHIDUME AND H. ZEGEYE
}

(Communicated by Joseph A. Ball)

\begin{abstract}
Let $K$ be a nonempty closed convex subset of a real Banach space $E$ and $T$ be a Lipschitz pseudocontractive self-map of $K$ with $F(T):=\{x \in$ $K: T x=x\} \neq \emptyset$. An iterative sequence $\left\{x_{n}\right\}$ is constructed for which $\left\|x_{n}-T x_{n}\right\| \rightarrow 0$ as $n \rightarrow \infty$. If, in addition, $K$ is assumed to be bounded, this conclusion still holds without the requirement that $F(T) \neq \emptyset$. Moreover, if, in addition, $E$ has a uniformly Gâteaux differentiable norm and is such that every closed bounded convex subset of $K$ has the fixed point property for nonexpansive self-mappings, then the sequence $\left\{x_{n}\right\}$ converges strongly to a fixed point of $T$. Our iteration method is of independent interest.
\end{abstract}

\section{INTRODUCTION}

Let $E$ be a real Banach space with dual $E^{*}$. We denote by $J$ the normalized duality mapping from $E$ to $2^{E^{*}}$ defined by

$$
J x:=\left\{f^{*} \in E^{*}:\left\langle x, f^{*}\right\rangle=\|x\|^{2}=\left\|f^{*}\right\|^{2}\right\},
$$

where $\langle.,$.$\rangle denotes the generalized duality pairing. It is well known that if E^{*}$ is strictly convex, then $J$ is single-valued. In the sequel, we shall denote the singlevalued normalized duality map by $j$.

A mapping $T$ with domain $D(T)$ and range $R(T)$ in $E$ is called pseudocontractive if the inequality

$$
\|x-y\| \leq\|x-y+t((I-T) x-(I-T) y)\|
$$

holds for each $x, y \in D(T)$ and for all $t>0$. The operator $T$ is called Lipschitzian if there exists $L \geq 0$ such that $\|T x-T y\| \leq L\|x-y\| \forall x, y \in D(T)$. If $L=1$, then $T$ is called nonexpansive. As a result of Kato [1], it follows from inequality (1.1) that $T$ is pseudocontractive if and only if there exists $j(x-y) \in J(x-y)$ such that $\langle T x-T y, j(x-y)\rangle \leq\|x-y\|^{2}$ for $x, y \in D(T)$. Clearly, every nonexpansive map is pseudocontractive.

Received by the editors May 27, 2002 and, in revised form, November 4, 2002.

2000 Mathematics Subject Classification. Primary 47H06, 47H09, 47J05, 47J25.

Key words and phrases. Normalized duality maps, uniformly Gâteaux differentiable norm, pseudocontractive maps.

The second author undertook this work when he was visiting the Abdus Salam International Centre for Theoretical Physics, Trieste, Italy, as a postdoctoral fellow. 
Apart from being an important generalization of nonexpansive maps, interest in pseudocontractive maps stems mainly from their firm connection with the important class of nonlinear accretive operators, where a mapping $A$ with domain $D(A)$ and range $R(A)$ in $E$ is called accretive if the inequality

$$
\|x-y\| \leq\|x-y+s(A x-A y)\|
$$

holds for every $x, y \in D(A)$ and for all $s>0$. $A$ is called $m$-accretive if it is accretive and $R(I+r A)$, the range of $(I+r A)$, is $E$ for all $r>0$. We observe that $A$ is accretive if and only if $T:=I-A$ is pseudocontractive and thus a zero of $A$ is a fixed point of $T$. It is now well known (see e.g., 27) that if $A$ is accretive, then the solutions of the equation $A x=0$ correspond to the equilibrium points of some evolution systems. Consequently, considerable research efforts, especially within the past 15 years or so, have been devoted to iterative methods for approximating fixed points of $T$ when $T$ is pseudocontractive (see for example [4], [14], 18], [21], [23], 28, and the references contained therein).

Let $T: K \rightarrow K$ be a nonexpansive self-mapping on a convex subset $K$ of a normed linear space $E$. Let $S_{\lambda}:=\lambda I+(1-\lambda) T, \lambda \in(0,1)$, where $I$ denotes the identity map of $K$. Then for fixed $x_{0} \in K,\left\{S_{\lambda}^{n}\left(x_{0}\right)\right\}$ is defined by $S_{\lambda}^{n}\left(x_{0}\right):=$ $\lambda x_{n}+(1-\lambda) T x_{n}$, where $x_{n}:=S_{\lambda}^{n-1}\left(x_{0}\right)$. In 1955, Krasnoselskii 12 proved that if $E$ is uniformly convex and $K$ is compact, then for any $x_{0} \in K$, the sequence $\left\{S_{\frac{1}{2}}^{n}\left(x_{0}\right)\right\}$ of iterates of $x_{0}$ under $S_{\frac{1}{2}}:=\frac{1}{2}(I+T)$ converges to a fixed point of $T$. Schaefer 24 observed that the same result holds for any $S_{\lambda}$ with $\lambda \in(0,1)$, and Edelstein [8] proved that strict convexity of $E$ suffices. The important and natural question of whether strict convexity can be removed remained open for many years. In 1976, this question was resolved in the affirmative in the following theorem.

Theorem I (10). Let $K$ be a nonempty subset of a Banach space $E$ and let $T: K \rightarrow E$ be a nonexpansive mapping. For $x_{0} \in K$, define the sequence $\left\{x_{n}\right\}$ by

$$
x_{n+1}:=\left(1-c_{n}\right) x_{n}+c_{n} T x_{n},
$$

where the real sequence $\left\{c_{n}\right\}$ satisfies the following conditions: (a) $\sum_{n=0}^{\infty} c_{n}=\infty$, (b) $0 \leq c_{n} \leq 1$ for all positive integers $n$; and (c) $x_{n} \in K$ for all positive integers $n$. If $\left\{x_{n}\right\}$ is bounded, then $\lim _{n \rightarrow \infty}\left\|x_{n}-T x_{n}\right\|=0$.

The iteration method of Theorem I is now referred to as the Mann iteration method in light of 14 and has been studied extensively by various authors. One consequence of this theorem is that if $K$ is closed and $T$ is completely continuous, then $T$ has a fixed point and the sequence $\left\{x_{n}\right\}$ defined by (1.3) converges strongly to a fixed point of $T$ (see, for example, Theorem 1 of [10]). Any sequence satisfying the conclusion of Theorem I, i.e., $\lim _{n \rightarrow \infty}\left\|x_{n}-T x_{n}\right\|=0$, is called an approximate fixed point sequence for $T$.

The importance of approximate fixed point sequences is that once a sequence has been constructed and proved to be an appropriate fixed point sequence for a nonexpansive map $T$, convergence of that sequence to a fixed point of $T$ is then achieved under some mild compactness-type assumptions either on $T$ or on its domain: for example, convergence to a fixed point can be proved under any of the following additional assumptions: (a) the range of $T$ is contained in a compact subset of $K$, (b) $T$ maps $K$ into $K$ and is demicompact at zero (see, e.g., [19]), 
(c) $(I-T)$ maps closed bounded subsets of $E$ into closed subsets of $E$ (see, e.g., [2]). These results are now well known for nonexpansive maps. But for classes of nonlinear maps $T$ more general than the class of nonexpansive maps, the condition $\left\|x_{n}-T x_{n}\right\| \rightarrow 0$ as $n \rightarrow \infty$ is generally part of the hypotheses in several convergence theorems (see, e.g., [13, 22]).

Our concern now is the following: Is it possible to extend Theorem I to the case where $T$ is a Lipschitz pseudocontractive map? In this connection, one of the authors and Mutangadura [6] have recently given an example of a Lipschitz pseudocontractive self-map of a compact convex subset of a Hilbert space with a unique fixed point for which no Mann sequence converges. Consequently, for this class of maps, the Mann sequence cannot give the conclusion of Theorem I. This now leads to the following important question.

Question 1. Is it possible to construct a perturbation of the recursion formula (1.3) or to construct another iteration sequence which yields the conclusion of Theorem I for the much more general and important class of Lipschitz pseudocontractive maps?

In 1974, Ishikawa 9 introduced an iteration process which, in some sense, is more general than that of Mann and which converges to a fixed point of a Lipschitzian pseudocontractive self-map $T$ of $K$. He proved the following theorem.

Theorem IS (9]). If $K$ is a compact convex subset of a Hilbert space $H, T$ : $K \mapsto K$ is a Lipschitzian pseudocontractive map and $x_{0}$ is any point of $K$, then the sequence $\left\{x_{n}\right\}_{n \geq 0}$ converges strongly to a fixed point of $T$, where $x_{n}$ is defined iteratively for each integer $n \geq 0$ by

$$
x_{n+1}=\left(1-\alpha_{n}\right) x_{n}+\alpha_{n} T y_{n} ; y_{n}=\left(1-\beta_{n}\right) x_{n}+\beta_{n} T x_{n},
$$

where $\left\{\alpha_{n}\right\},\left\{\beta_{n}\right\}$ are sequences of positive numbers satisfying the conditions

$$
\text { (i) } 0 \leq \alpha_{n} \leq \beta_{n}<1 ; \quad \text { (ii) } \lim _{n \rightarrow \infty} \beta_{n}=0 ; \quad \text { (iii) } \sum_{n \geq 0} \alpha_{n} \beta_{n}=\infty \text {. }
$$

The iteration method of Theorem IS, which is now referred to as the Ishikawa iteration method, has been studied extensively by various authors. However, it is still an open question whether or not this method can be employed to approximate fixed points of Lipschitz pseudocontractive maps in spaces more general than Hilbert spaces (see, e.g., [5], 20], 21]).

Another iteration method has also been introduced by Schu [25]. To state the method, we need the following definition.

Definition (see, e.g., 25]). Let $\alpha_{n} \in(0, \infty), \mu_{n} \in(0,1)$ for all nonnegative integers $n$. Then $\left(\left\{\alpha_{n}\right\},\left\{\mu_{n}\right\}\right)$ is said to have property (A) if and only if the following conditions hold:

(i) $\left\{\alpha_{n}\right\}$ is decreasing and $\left\{\mu_{n}\right\}$ is strictly increasing;

(ii) there is a sequence $\left\{\beta_{n}\right\} \subseteq \mathbb{N}$, strictly increasing such that (a) $\lim _{n \rightarrow \infty} \beta_{n}\left(1-\mu_{n}\right)=\infty$; (b) $\lim _{n \rightarrow \infty} \frac{1-\mu_{\left(n+\beta_{n}\right)}}{1-\mu_{n}}=1$; (c) $\lim _{n \rightarrow \infty} \frac{\alpha_{n}-\alpha_{\left(n+\beta_{n}\right)}}{1-\mu_{n}}=0$.

Schu proved the following theorem.

Theorem S (25]). Let $K$ be a nonempty closed bounded and convex subset of a Hilbert space $H$. Suppose that (i) $T: K \rightarrow K$ is pseudocontractive and Lipschitzian with $L \geq 0$; (ii) $\left\{\lambda_{n}\right\}_{n \in \mathbb{N}} \subset(0,1)$ with $\lim _{n \rightarrow \infty} \lambda_{n}=1,\left\{\alpha_{n}\right\}_{n \in \mathbb{N}} \subset(0,1)$ with 
$\lim _{n \rightarrow \infty} \alpha_{n}=0$, such that $\left(\left\{\alpha_{n}\right\},\left\{\mu_{n}\right\}\right)$ has property (A), $\left(\left(1-\mu_{n}\right)\left(1-\lambda_{n}\right)^{-1}\right)$ is bounded and $\lim _{n \rightarrow \infty} \alpha_{n}^{-1}\left(1-\mu_{n}\right)=0$, where $k_{n}=\left(1+\alpha_{n}^{2}(1+L)^{2}\right)^{\frac{1}{2}}$ and $\mu_{n}:=\lambda_{n} k_{n}^{-1}$ for all $n \in \mathbb{N}$. For arbitrary vectors $z_{0}, w \in K$, define, for all $n \in \mathbb{N}$,

$$
z_{n+1}=\left(1-\mu_{n+1}\right) w+\mu_{n+1} y_{n} ; y_{n}=\left(1-\alpha_{n}\right) z_{n}+\alpha_{n} T z_{n} .
$$

Then $\left\{z_{n}\right\}$ converges strongly to the unique fixed point of $T$ closest to $w$.

Unlike the Ishikawa method, Theorem $\mathrm{S}$ has been extended to real Banach spaces more general than Hilbert spaces. In [3], one of the authors extended it to real Banach spaces possessing weakly sequentially continuous duality maps (e.g., $l_{p}$ spaces, $1<p<\infty)$. In this extension the iteration parameters $\alpha_{n} \in(0, \infty)$ and $\mu_{n} \in(0,1)$ are chosen such that the pair $\left(\left\{\alpha_{n}\right\},\left\{\mu_{n}\right\}\right)$ has the so-called property (A). These choices are not simple (see, e.g., 3]). Moreover, it is known that $L_{p}$ spaces, $1<p<\infty, p \neq 2$ do not possess weakly sequentially continuous duality maps. This brings us to our second question.

Question 2. Can an iteration sequence be constructed which converges to a fixed point of a Lipschitz pseudocontractive map in Banach spaces that include the $L_{p}$ spaces, $1<p<\infty$ ?

It is our purpose in this paper to give affirmative answers to Question 1 and Question 2. Let $K$ be a nonempty closed convex subset of a real Banach space $E$ and $T$ be a Lipschitz pseudocontractive self-map of $K$ with $F(T) \neq \emptyset$, and let a sequence $\left\{x_{n}\right\}$ be generated from $x_{1} \in K$ by $x_{n+1}:=\left(1-\lambda_{n}\right) x_{n}+\lambda_{n} T x_{n}-\lambda_{n} \theta_{n}\left(x_{n}-x_{1}\right)$, for all integers $n \geq 1$, where $\left\{\lambda_{n}\right\}$ and $\left\{\theta_{n}\right\}$ are real sequences satisfying appropriate conditions. Then $\left\|x_{n}-T x_{n}\right\| \rightarrow 0$ as $n \rightarrow \infty$. This provides an affirmative answer to Question 1. If, in addition, $K$ is assumed to be bounded, this conclusion still holds without the requirement that $F(T) \neq \emptyset$. Moreover, if, in addition, $E$ has a uniformly Gâteaux differentiable norm and is such that every closed bounded convex subset of $K$ has the fixed point property for nonexpansive self-mappings (e.g., $L_{p}$ spaces, $1<p<\infty$ ), then $\left\{x_{n}\right\}$ converges strongly to a fixed point of $T$. This result provides an affirmative answer to Question 2. Our iteration method is of independent interest (see our Remark 3.8).

\section{Preliminaries}

Let $E$ be a real normed linear space of dimension $\geq 2$. The modulus of smoothness of $E$ is defined by

$$
\rho_{E}(\tau):=\sup \left\{\frac{\|x+y\|+\|x-y\|}{2}-1:\|x\|=1,\|y\|=\tau\right\} .
$$

A Banach space $E$ is called uniformly smooth if $\lim _{\tau \rightarrow 0} \frac{\rho_{E}(\tau)}{\tau}=0$. Typical examples of such spaces are the Lebesgue $L_{p}$, the sequence $\ell_{p}$ and the Sobolev $W_{p}^{m}$ spaces $(1<p<\infty)$. The norm is said to be uniformly Gâteaux differentiable if for each $y \in S_{1}(0):=\{x \in E:\|x\|=1\}$ the $\operatorname{limit}_{\lim } \frac{\|x+t y\|-\|x\|}{t}$ exists uniformly for $x \in S_{1}(0)$. It is well known that every uniformly smooth space (e.g., $L_{p}$ space, $1<p<\infty$ ) has uniformly Gâteaux differentiable norm (see, e.g., [7]).

Let $K$ be a nonempty subset of a Banach space $E$. For $x \in K$, the inward set of $x, I_{K}(x)$, is defined by $I_{K}(x):=\{x+\lambda(u-x): u \in K, \lambda \geq 1\}$. A mapping 
$T: K \rightarrow E$ is called weakly inward if $T x \in \operatorname{cl}\left[I_{K}(x)\right]$ for all $x \in K$, where $\operatorname{cl}\left[I_{K}(x)\right]$ denotes the closure of the inward set. Every self-map is trivially weakly inward.

In what follows, we shall make use of the following lemmas.

Lemma 2.1 (see, e.g., [17]). Let $E$ be a real normed linear space and $J$ the normalized duality map on $E$. Then for any given $x, y \in E$, the following inequality holds:

$$
\|x+y\|^{2} \leq\|x\|^{2}+2\langle y, j(x+y)\rangle, \forall j(x+y) \in J(x+y) .
$$

Lemma 2.2 (see [15]). Let $\left\{\lambda_{n}\right\},\left\{\alpha_{n}\right\}$ and $\left\{\gamma_{n}\right\}$ be sequences of nonnegative numbers satisfying the conditions $\lim \alpha_{n}=0, \sum_{1}^{\infty} \alpha_{n}=\infty$, and $\frac{\gamma_{n}}{\alpha_{n}} \rightarrow 0$, as $n \rightarrow \infty$. Let the recursive inequality

$$
\lambda_{n+1}^{2} \leq \lambda_{n}^{2}-\alpha_{n} \psi\left(\lambda_{n+1}\right)+\gamma_{n}, \quad n=1,2, \ldots,
$$

be given where $\psi:[0, \infty) \rightarrow[0, \infty)$ is a strictly increasing function such that it is positive on $(0, \infty)$ and $\psi(0)=0$. Then $\lambda_{n} \rightarrow 0$ as $n \rightarrow \infty$.

Lemma MJ (Proposition 1, 17). Let $E$ be a Banach space. Suppose $K$ is a nonempty closed convex subset of $E$ and $T: K \rightarrow E$ is a continuous pseudocontractive mapping satisfying the weakly inward condition. Then for $z \in K$, there exists a unique path $t \rightarrow y_{t} \in K, t \in[0,1)$, satisfying the following condition:

$$
y_{t}=t T y_{t}+(1-t) z \text {. }
$$

We note that in Lemma MJ if, in addition, $F(T) \neq \emptyset$, then $\left\{y_{t}\right\}$ is bounded. Furthermore, if $E$ is assumed to have a uniformly Gâteaux differentiable norm and is such that every closed convex and bounded subset of $K$ has the fixed point property for nonexpansive self-mappings, then as $t \rightarrow 1$, the path converges strongly to a fixed point of $T$ (see [17]).

\section{MAin Results}

For the rest of this paper, $\left\{\lambda_{n}\right\}$ and $\left\{\theta_{n}\right\}$ are real sequences in $(0,1]$ satisfying the following conditions: (i) $\lim _{n \rightarrow \infty} \theta_{n}=0$; (ii) $\lambda_{n}\left(1+\theta_{n}\right) \leq 1, \sum \lambda_{n} \theta_{n}=\infty$,

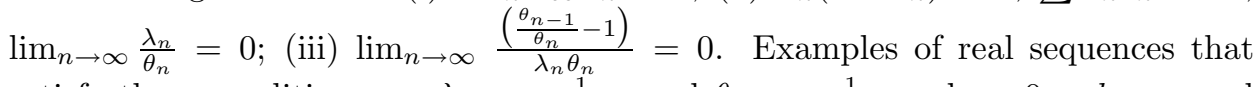
satisfy these conditions are $\lambda_{n}=\frac{1}{(n+1)^{a}}$ and $\theta_{n}=\frac{1}{(n+1)^{b}}$, where $0<b<a$ and $a+b<1$. Also $\left\{y_{n}\right\}$ denotes the sequence defined by $y_{n}:=y_{t_{n}}=t_{n} T y_{t_{n}}+(1-$ $\left.t_{n}\right) x_{1}, t_{n}=\frac{1}{1+\theta_{n}}, \forall n \geq 1$, guaranteed by Lemma MJ.

We now prove the following theorems.

Theorem 3.1. Let $K$ be a nonempty closed convex subset of a real Banach space $E$. Let $T: K \rightarrow K$ be a Lipschitz pseudocontractive map with Lipschitz constant $L \geq 0$ and $F(T) \neq \emptyset$. Let a sequence $\left\{x_{n}\right\}$ be generated from arbitrary $x_{1} \in K$ by

$$
x_{n+1}:=\left(1-\lambda_{n}\right) x_{n}+\lambda_{n} T x_{n}-\lambda_{n} \theta_{n}\left(x_{n}-x_{1}\right),
$$

for all positive integers $n$. Then $\left\|x_{n}-T x_{n}\right\| \rightarrow 0$ as $n \rightarrow \infty$.

Proof. Since $\frac{\lambda_{n}}{\theta_{n}} \rightarrow 0$ there exists $N_{0}>0$ such that $\frac{\lambda_{n}}{\theta_{n}} \leq d:=\frac{1}{2\left(\frac{5}{2}+L\right)(2+L)}, \forall n \geq$ $N_{0}$. Let $x^{*} \in F(T)$ and $r>0$ be sufficiently large such that $x_{N_{0}} \in B_{r}\left(x^{*}\right)$ and $x_{1} \in B_{\frac{r}{2}}\left(x^{*}\right)$. We split the proof into three parts.

(i) We prove $\left\{x_{n}\right\}$ is bounded. It suffices to show by induction that $\left\{x_{n}\right\}$ belongs to $B:=\overline{B_{r}\left(x^{*}\right)}$ for all integers $n \geq N_{0}$. Now, $x_{N_{0}} \in B$ by construction. Hence we 
may assume $x_{n} \in B$ for any $n>N_{0}$ and prove that $x_{n+1} \in B$. Suppose $x_{n+1}$ is not in $B$. Then $\left\|x_{n+1}-x^{*}\right\|>r$ and thus from the recursion formula (3.1) and Lemma 2.1 we get that

$$
\begin{aligned}
\left\|x_{n+1}-x^{*}\right\|^{2}= & \left\|x_{n}-x^{*}-\lambda_{n}\left(\left(x_{n}-T x_{n}\right)+\theta_{n}\left(x_{n}-x_{1}\right)\right)\right\|^{2} \\
\leq & \left\|x_{n}-x^{*}\right\|^{2}-2 \lambda_{n}\left\langle\left(x_{n}-T x_{n}\right)+\theta_{n}\left(x_{n}-x_{1}\right), j\left(x_{n+1}-x^{*}\right)\right\rangle \\
= & \left\|x_{n}-x^{*}\right\|^{2}-2 \lambda_{n} \theta_{n}\left\|x_{n+1}-x^{*}\right\|^{2} \\
& +2 \lambda_{n}\left\langle\theta_{n}\left(x_{n+1}-x_{n}\right)-\left(x_{n}-T x_{n}\right)+\theta_{n}\left(x_{1}-x^{*}\right)\right. \\
& \left.+\left(x_{n+1}-T x_{n+1}\right)-\left(x_{n+1}-T x_{n+1}\right), j\left(x_{n+1}-x^{*}\right)\right\rangle .
\end{aligned}
$$

Since $T$ is pseudocontractive we have $\left\langle x_{n+1}-T x_{n+1}, j\left(x_{n+1}-x^{*}\right)\right\rangle \geq 0$. Thus, (3.2) gives

$$
\begin{aligned}
\left\|x_{n+1}-x^{*}\right\|^{2} \leq & \left\|x_{n}-x^{*}\right\|^{2}-2 \lambda_{n} \theta_{n}\left\|x_{n+1}-x^{*}\right\|^{2} \\
& +2 \lambda_{n}\left\langle\theta_{n}\left(x_{n+1}-x_{n}\right)+\theta_{n}\left(x_{1}-x^{*}\right)\right. \\
& \left.+\left(x_{n+1}-T x_{n+1}\right)-\left(x_{n}-T x_{n}\right), j\left(x_{n+1}-x^{*}\right)\right\rangle \\
\leq \quad & \left\|x_{n}-x^{*}\right\|^{2}-2 \lambda_{n} \theta_{n}\left\|x_{n+1}-x^{*}\right\|^{2} \\
& +2 \lambda_{n}\left[(2+L)\left\|x_{n+1}-x_{n}\right\|+\theta_{n}\left\|x_{1}-x^{*}\right\|\right]\left\|x_{n+1}-x^{*}\right\| \\
= & \left\|x_{n}-x^{*}\right\|^{2}-2 \lambda_{n} \theta_{n}\left\|x_{n+1}-x^{*}\right\|^{2} \\
& +2 \lambda_{n}\left[(2+L) \lambda_{n}\left\|\left(x_{n}-T x_{n}\right)+\theta_{n}\left(x_{n}-x_{1}\right)\right\|\right. \\
& \left.+\theta_{n}\left\|x_{1}-x^{*}\right\|\right]\left\|x_{n+1}-x^{*}\right\| \\
\leq \quad & \left\|x_{n}-x^{*}\right\|^{2}-2 \lambda_{n} \theta_{n}\left\|x_{n+1}-x^{*}\right\|^{2} \\
& +2 \lambda_{n}\left[(2+L) \lambda_{n}\left((2+L)\left\|x_{n}-x^{*}\right\|+\theta_{n}\left\|x_{1}-x^{*}\right\|\right)\right. \\
& \left.+\theta_{n}\left\|x_{1}-x^{*}\right\|\right]\left\|x_{n+1}-x^{*}\right\| \\
\leq \quad & \left\|x_{n}-x^{*}\right\|^{2}-2 \lambda_{n} \theta_{n}\left\|x_{n+1}-x^{*}\right\|^{2} \\
& +2 \lambda_{n}\left[\lambda_{n}(2+L)\left(\frac{5}{2}+L\right) r+\frac{\theta_{n}}{2} r\right]\left\|x_{n+1}-x^{*}\right\|,
\end{aligned}
$$

since $x_{n} \in B$ and $x_{1} \in B_{\frac{r}{2}}\left(x^{*}\right)$. However, $\left\|x_{n+1}-x^{*}\right\|>\left\|x_{n}-x^{*}\right\|$. So we have from (3.3) that

$$
\theta_{n}\left\|x_{n+1}-x^{*}\right\| \leq\left[\lambda_{n}(2+L)\left(\frac{5}{2}+L\right) r+\frac{\theta_{n}}{2} r\right]
$$

and hence $\left\|x_{n+1}-x^{*}\right\| \leq r$, since $\frac{\lambda_{n}}{\theta_{n}} \leq \frac{1}{2\left(\frac{5}{2}+L\right)(2+L)}, \forall n \geq N_{0}$. Thus we get a contradiction. Therefore, $x_{n} \in B$ for all positive integers $n \geq N_{0}$ and hence the sequence $\left\{x_{n}\right\}$ is bounded.

(ii) We prove $\left\|x_{n}-y_{n}\right\| \rightarrow 0$ as $n \rightarrow \infty$. From the recursion formula (3.1) and Lemma 2.1 we have that

$$
\begin{aligned}
\left\|x_{n+1}-y_{n}\right\|^{2} \leq & \left\|x_{n}-y_{n}\right\|^{2}-2 \lambda_{n} \theta_{n}\left\langle\left(x_{n+1}-y_{n}\right), j\left(x_{n+1}-y_{n}\right)\right\rangle \\
& +2 \lambda_{n}\left\langle\theta_{n}\left(x_{n+1}-y_{n}\right)-\left(x_{n}-T x_{n}\right)-\theta_{n}\left(x_{n}-x_{1}\right), j\left(x_{n+1}-y_{n}\right)\right\rangle \\
= & \left\|x_{n}-y_{n}\right\|^{2}-2 \lambda_{n} \theta_{n}\left\|x_{n+1}-y_{n}\right\|^{2}+2 \lambda_{n}\left\langle\theta_{n}\left(x_{n+1}-x_{n}\right)\right. \\
& +\left[\theta_{n}\left(x_{1}-y_{n}\right)-\left(y_{n}-T y_{n}\right)\right]-\left[\left(x_{n+1}-T x_{n+1}\right)-\left(y_{n}-T y_{n}\right)\right] \\
& \left.+\left[\left(x_{n+1}-T x_{n+1}\right)-\left(x_{n}-T x_{n}\right)\right], j\left(x_{n+1}-y_{n}\right)\right\rangle .
\end{aligned}
$$


Observe that by the property of $y_{n}$ and pseudocontractivity of $T$ we have that $\theta_{n}\left(x_{1}-y_{n}\right)-\left(y_{n}-T y_{n}\right)=0$ and $\left\langle\left(x_{n+1}-T x_{n+1}\right)-\left(y_{n}-T y_{n}\right), j\left(x_{n+1}-y_{n}\right)\right\rangle \geq 0$ for all $n \geq 1$. Thus, we have from (3.4) that

$$
\begin{aligned}
\left\|x_{n+1}-y_{n}\right\|^{2} \leq & \left\|x_{n}-y_{n}\right\|^{2}-2 \lambda_{n} \theta_{n}\left\|x_{n+1}-y_{n}\right\|^{2}+2 \lambda_{n}\left\langle\theta_{n}\left(x_{n+1}-x_{n}\right)\right. \\
& \left.+\left[\left(x_{n+1}-T x_{n+1}\right)-\left(x_{n}-T x_{n}\right)\right], j\left(x_{n+1}-y_{n}\right)\right\rangle \\
\leq & \left\|x_{n}-y_{n}\right\|^{2}-2 \lambda_{n} \theta_{n}\left\|x_{n+1}-y_{n}\right\|^{2} \\
& +2 \lambda_{n}(2+L)\left\|x_{n+1}-x_{n}\right\| \cdot\left\|x_{n+1}-y_{n}\right\| \\
\leq & \left\|x_{n}-y_{n}\right\|^{2}-2 \lambda_{n} \theta_{n}\left\|x_{n+1}-y_{n}\right\|^{2} \\
& +2 \lambda_{n}^{2}(2+L)\left\|x_{n}-T x_{n}+\theta_{n}\left(x_{n}-x_{1}\right)\right\| .\left\|x_{n+1}-y_{n}\right\| .
\end{aligned}
$$

However, since $F(T) \neq \emptyset$, by Proposition 2 of [17] we have that $\left\{y_{n}\right\}$ is bounded. Therefore, there exists $M_{1}>0$ such that $\max \left\{\left\|x_{n+1}-y_{n}\right\|, \| x_{n}-T x_{n}+\theta_{n}\left(x_{n}-\right.\right.$ $\left.\left.x_{1}\right) \|\right\} \leq M_{1}$. Thus from (3.5) we get that

$$
\left\|x_{n+1}-y_{n}\right\|^{2} \leq\left\|x_{n}-y_{n}\right\|^{2}-2 \lambda_{n} \theta_{n}\left\|x_{n+1}-y_{n}\right\|^{2}+2 \lambda_{n}^{2}(2+L) M_{1} .
$$

Moreover, by the pseudocontractivity of $T$ we have that

$$
\begin{aligned}
\left\|y_{n-1}-y_{n}\right\| & \leq\left\|y_{n-1}-y_{n}+\frac{1}{\theta_{n}}\left(\left(y_{n-1}-T y_{n-1}\right)-\left(y_{n}-T y_{n}\right)\right)\right\| \\
& \leq \frac{\theta_{n-1}-\theta_{n}}{\theta_{n}}\left(\left\|y_{n-1}\right\|+\left\|x_{1}\right\|\right)=\left(\frac{\theta_{n-1}}{\theta_{n}}-1\right)\left(\left\|y_{n-1}\right\|+\left\|x_{1}\right\|\right) .
\end{aligned}
$$

Thus, from (3.6) and (3.7) we get that

$$
\begin{aligned}
\left\|x_{n+1}-y_{n}\right\|^{2} \leq & \left\|x_{n}-y_{n-1}\right\|^{2}-2 \lambda_{n} \theta_{n}\left\|x_{n+1}-y_{n}\right\|^{2} \\
& +M\left(\frac{\theta_{n-1}}{\theta_{n}}-1\right)+2 \lambda_{n}^{2}(2+L) M,
\end{aligned}
$$

for some constant $M>0$. By Lemma 2.2 and the conditions on $\left\{\lambda_{n}\right\}$ and $\left\{\theta_{n}\right\}$ we get $x_{n+1}-y_{n} \rightarrow 0$. Consequently, $\left\|x_{n}-y_{n}\right\| \rightarrow 0$ as $n \rightarrow \infty$.

(iii) We prove $\left\|x_{n}-T x_{n}\right\| \rightarrow 0$ as $n \rightarrow \infty$. Since $\left\{y_{n}\right\}$ (and hence $\left\{T y_{n}\right\}$ ) is bounded we have $\left\|y_{n}-T y_{n}\right\| \leq\left(1-t_{n}\right)\left\|T y_{n}\right\|+\left(1-t_{n}\right)\left\|x_{1}\right\| \rightarrow 0$. Hence

$$
\begin{aligned}
\left\|x_{n}-T x_{n}\right\| & \leq\left\|x_{n}-y_{n}\right\|+\left\|y_{n}-T y_{n}\right\|+\left\|T y_{n}-T x_{n}\right\| \\
& \leq(1+L)\left\|x_{n}-y_{n}\right\|+\left\|y_{n}-T y_{n}\right\| \rightarrow 0, \text { as } n \rightarrow \infty .
\end{aligned}
$$

This completes the proof of the theorem.

Remark 3.2. Theorem 3.1 provides an appropriate fixed point sequence for a Lipschitz pseudocontractive map in a real Banach space, thus resolving Question 1 in the affirmative.

Theorem 3.3. Let $K$ be a nonempty closed convex subset of a real Banach space $E$ with a uniformly Gâteaux differentiable norm. Let $T: K \rightarrow K$ be a Lipschitz pseudocontractive map with Lipschitz constant $L \geq 0$ and $F(T) \neq \emptyset$. Suppose every closed convex and bounded subset of $K$ has the fixed point property for nonexpansive self-mappings. Let a sequence $\left\{x_{n}\right\}$ be generated from arbitrary $x_{1} \in K$ by

$$
x_{n+1}:=\left(1-\lambda_{n}\right) x_{n}+\lambda_{n} T x_{n}-\lambda_{n} \theta_{n}\left(x_{n}-x_{1}\right),
$$

for all positive integers $n$. Then $\left\{x_{n}\right\}$ converges strongly to a fixed point of $T$.

Proof. As in the proof of Theorem 3.1 we get that $\left\|x_{n}-y_{n}\right\| \rightarrow 0$ as $n \rightarrow \infty$, and by Theorem 1 of [17] we have that $y_{n} \rightarrow x^{*} \in F(T)$. The conclusion follows. 
Corollary 3.4. Let $E$ be a real Banach space with a uniformly Gâteaux differentiable norm. Let $A: E \rightarrow E$ be a Lipschitzian accretive operator with Lipschitz constant $L^{\prime} \geq 0$ and $N(A) \neq \emptyset$, where $N(A):=\{x \in E: A x=0\}$. Suppose every closed convex and bounded subset of $E$ has the fixed point property for nonexpansive self-mappings. Let a sequence $\left\{x_{n}\right\}$ be generated from arbitrary $x_{1} \in E$ by

$$
x_{n+1}=x_{n}-\lambda_{n} A x_{n}-\lambda_{n} \theta_{n}\left(x_{n}-x_{1}\right),
$$

for all positive integers $n$. Then $\left\{x_{n}\right\}$ converges strongly to a solution of the equation $A x=0$.

Proof. Since $T:=(I-A)$ is a Lipschitz pseudocontractive map with Lipschitz constant $L:=\left(L^{\prime}+1\right)$ and the fixed point of $T$ is the solution of the equation $A x=0$, the conclusion follows from Theorem 3.3.

Corollary 3.5. Let $K$ be a nonempty closed convex subset of a real uniformly smooth Banach space $E$. Let $T: K \rightarrow K$ be a Lipschitz pseudocontractive map with Lipschitz constant $L \geq 0$ and $F(T) \neq \emptyset$. Let a sequence $\left\{x_{n}\right\}$ be generated from arbitrary $x_{0} \in K$ by

$$
x_{n+1}=\left(1-\lambda_{n}\right) x_{n}+\lambda_{n} T x_{n}-\lambda_{n} \theta_{n}\left(x_{n}-x_{1}\right),
$$

for all positive integers $n$. Then $\left\{x_{n}\right\}$ converges strongly to a fixed point of $T$.

Proof. Since every uniformly smooth Banach space has uniformly Gâteaux differentiable norm and every closed bounded convex subset of $K$ has the fixed point property for nonexpansive self-mappings (see, e.g., [26]), the conclusion follows from Theorem 3.3.

Remark 3.6. In Theorem 3.1, if in addition $K$ is bounded, then by Proposition 2 of [17], the sequence $\left\{y_{n}\right\}$ is bounded. Therefore, the condition that $F(T) \neq \emptyset$ will not be required in the proof. Hence, we have the conclusions of Theorem 3.3 and Corollary 3.5 without the assumption that $F(T) \neq \emptyset$.

Remark 3.7. Theorem 3.3 and Corollaries 3.4 and 3.5 provide convergence results for fixed points of Lipschitz pseudocontractive maps (or for zeros of accretive maps) in real Banach spaces much more general than Hilbert spaces. In particular, Theorem 3.3 and Corollary 3.5 independently provide an affirmative answer to Question 2 .

Remark 3.8. We first remark that the existence of a path for Lipschitz pseudocontractive maps was first established by Morales [16]. We also note that Bruck [1] studied the recursion formula (3.1) for approximating solutions of the equation $A u=0$ in a Hilbert space, where $A$ is an $m$-accretive operator. He required that $\lambda_{n}$ and $\theta_{n}$ be acceptably paired sequences, i.e., that they satisfy the following conditions: $\left\{\theta_{n}\right\}$ is decreasing, $\lim _{n \rightarrow \infty} \theta_{n}=0$ and there exists a strictly increasing sequence $\{n(i)\}_{i=1}^{\infty}$ of positive integers such that (i) $\liminf _{i} \theta_{n(i)} \sum_{j=n(i)}^{n(i+1)} \lambda_{j}>0$, (ii) $\lim _{i}\left[\theta_{n(i)}-\theta_{n(i+1)}\right] \sum_{j=n(i)}^{n(i+1)} \lambda_{j}=0$, and (iii) $\lim _{i} \sum_{j=n(i)}^{n(i+1)} \lambda_{j}^{2}=0$. An example of acceptably paired sequences is $\lambda_{n}=n^{-1}, \theta_{n}=(\log \log n)^{-1}, n(i)=i^{i}$ (see, e.g., [1]). Reich [23] also studied the recursion formula (3.1) for Lipschitz accretive operators 
on real uniformly convex Banach spaces with a duality mapping that is weakly sequentially continuous at zero and with $\lambda_{n}$ and $\theta_{n}$ satisfying conditions slightly stronger than our conditions as given at the beginning of Section 3.

A comparison of formula (3.1) with $\lambda_{n}$ and $\theta_{n}$ being acceptably paired, formulas (1.4) in which $\alpha_{n}$ and $\beta_{n}$ can be chosen as $\alpha_{n}=\beta_{n}=\left(1+\beta_{n}\right)^{-\frac{1}{2}}$ for all integers $n \geq 0$, and formulas (1.5) with $\left(\left\{\alpha_{n}\right\},\left\{\mu_{n}\right\}\right)$ having the so-called property $(A)$, all of which have been used to approximate fixed points of Lipschitz pseudocontractive maps in Hilbert spaces, shows that formulas (1.4) are, perhaps, the best because of the ease of the choices of $\alpha_{n}$ and $\beta_{n}$. This probably is the reason for the interest in and extensive study of the Ishikawa iteration method.

But now, in our study in this paper of the recursion formula (3.1), our technique of proof makes it possible for us to dispense with the requirement that $\lambda_{n}$ and $\theta_{n}$ be acceptably paired sequences. As has been noted at the beginning of Section 3, $\lambda_{n}$ and $\theta_{n}$ can now be chosen as easily as $\alpha_{n}$ and $\beta_{n}$ are chosen in the Ishikawa method. Moreover, formula (3.1) requires less computer time than formula (1.4) of the Ishikawa method. Furthermore, formula (3.1) allows the approximation of fixed points of Lipschitz pseudocontractive maps (when they exist) in real Banach spaces much more general than Hilbert spaces. Given these advantages of formula (3.1) over formulas (1.4), it is clear that the iteration method studied in this paper is superior to the Ishikawa iteration method that has been studied extensively in recent years by various authors.

\section{REFERENCES}

[1] R. E. Bruck, Jr.; A strongly convergent iterative method for the solution of $0 \in U(x)$ for a maximal monotone operator $U$ in Hilbert space, J. Math. Anal. Appl. 48 (1974), 114-126. MR 50:14383

[2] C. E. Chidume; On the approximation of fixed points of nonexpansive mappings, Houston J. Math. 7 (1981), 345-355. MR 83b:47063

[3] C. E. Chidume; Iterative approximation of fixed points of Lipschitz pseudocontractive maps, Proc. Amer. Math. Soc. 129 (2001), 2245-2251. MR 2002e:47071

[4] C. E. Chidume and C. Moore; The solution by iteration of nonlinear equations in uniformly smooth Banach spaces, J. Math. Anal. Appl. 215 (1997), 132-146. MR 98m:47107

[5] C. E. Chidume and C. Moore; Fixed point iteration for pseudocontractive maps, Proc. Amer. Math. Soc. 127(4) (1999), 1163-1170. MR 99f:47068

[6] C. E. Chidume and S. Mutangadura; An example on the Mann iteration method for Lipschitz pseudocontractions, Proc. Amer. Math. Soc. 129 (2001), (8), 2359-2363. MR 2002f:47104

[7] I. Cioranescu; Geometry of Banach spaces, duality mappings and nonlinear problems, Kluwer Academic Publishers, Dordrecht, 1990. MR 91m:46021

[8] M. Edelstein; A remark on a theorem of M. A. Krasnoselskii, Amer. Math. Monthly 73 (1966), 509-510. MR 33:3072

[9] S. Ishikawa; Fixed points by a new iteration method, Proc. Amer. Math. Soc. 44(1) (1974), 147-150. MR 49:1243

[10] S. Ishikawa; Fixed points and iteration of a nonexpansive mapping in a Banach space, Proc. Amer. Math. Soc. 59(1) (1976), 65-71. MR 54:1030

[11] T. Kato; Nonlinear semigroups and evolution equations, J. Math. Soc. Japan 19 (1967), 508-520. MR 37:1820

[12] M. A. Krasnoselskii; Two remarks on the method of successive approximations, Uspehi Mat. Nauk 10 (1955), 123-127. MR 16:833a

[13] T. C. Lim and H. K. Xu; Fixed point theorems for asymptotically nonexpansive mappings, Nonlinear Anal. TMA 22 (1994), 1345-1355. MR 95i:47103

[14] W. R. Mann; Mean value methods in iteration, Proc. Amer. Math. Soc. 4 (1953), 506-510. MR 14:988f 
[15] C. Moore and B.V.C. Nnoli; Iterative solution of nonlinear equations involving set-valued uniformly accretive operators, Comput. Math. Appl. 42 (2001), 131-140. MR 2002f:47145

[16] C. H. Morales, Strong convergence theorems for pseudo-contractive mappings in Banach spaces, Houston J. Math. 16 (1990), 549-557. MR 92f:47063

[17] C. H. Morales and J. S. Jung; Convergence of paths for pseudocontractive mappings in Banach spaces, Proc. Amer. Math. Soc. 128 (2000), 3411-3419. MR 2001b:47090

[18] M. O. Osilike; Iterative solution of nonlinear equations of the $\psi$-strongly accretive type, $J$. Math. Anal. Appl. 200 (1996), 259-271. MR 97d:65032

[19] W. V. Petryshyn; Construction of fixed points of demicompact mappings in Hilbert space, $J$. Math. Anal. Appl. 14 (1966), 276-284. MR 33:3147

[20] L. Qihou; On Naimpally and Singh's open questions, J. Math. Anal. Appl. 124 (1987), 157164. MR 88j: 47078

[21] L. Qihou; The convergence theorems of the sequence of Ishikawa iterates for hemicontractive mappings, J. Math. Anal. Appl. 148 (1990), 55-62. MR 92b:47094

[22] L. Qihou; Iterative sequences for asymptotically quasi-nonexpansive mappings, J. Math. Anal. Appl. 259 (2001), 1-7. MR 2002h:47111

[23] S. Reich; Iterative methods for accretive sets, in "Nonlinear Equations in Abstract Spaces", Academic Press, New York, 1978, pp. 317-326. MR 82c:47065

[24] H. Schaefer; Uber die Methode sukzessiver Approximationen, Jber. Deutsch. Math. Verein. 59 (1957), 131-140. MR 18:811g

[25] J. Schu; Approximating fixed points of Lipschitzian pseudocontractive mappings, Houston J. Math. 19 (1993), 107-115. MR 94f:47066

[26] B. Turett; A dual view of a theorem of Baillon, Nonlinear Analysis and Applications (S .P. Singh and J. H. Burry, eds.), Marcel Dekker, New York, 1982, pp. 279-286. MR 84g:47054

[27] E. Zeidler; Nonlinear Functional Analysis and its Applications, Part IIA: Linear Monotone Operators; Part IIB: Nonlinear Monotone Operators (Springer-Verlag, Berlin/New York, 1985). MR 91b:47001, MR 91b:47002

[28] S. Zhang; On the convergence problems of Ishikawa and Mann iteration processes with error for $\psi$-pseudo contractive type mappings. Appl. Math. Mechanics 21 (2000), 1-12. MR 2001c: 47080

The Abdus Salam International Centre for Theoretical Physics, Trieste, Italy

E-mail address: chidume@ictp.trieste.it

The Abdus Salam International Centre for Theoretical Physics, Trieste, Italy

E-mail address: habz@ictp.trieste.it 